\title{
SPECTRAL INTEGRATION AND TWO-POINT BOUNDARY VALUE PROBLEMS*
}

\author{
L. GREENGARD $\dagger$
}

\begin{abstract}
A numerical method for two-point boundary value problems with constant coefficients is developed which is based on integral equations and the spectral integration matrix for Chebyshev nodes. The method is stable, achieves superalgebraic convergence, and requires $O(N \log N)$ operations, where $N$ is the number of nodes in the discretization. Although stable spectral methods have been constructed in the past, they have generally been based on reformulating the recurrence relations obtained through spectral differentiation in an attempt to avoid the ill-conditioning introduced by that process.
\end{abstract}

Key words. spectral methods, integral equations, two-point boundary value problems

AMS(MOS) subject classifications. 65D30, 65L10, 34B05, 45L10

1. Introduction. Spectral methods are now a popular tool for the solution of many types of partial differential equations. In the usual formulation, the basic idea is to represent the solution $f$ by means of a truncated series expansion, and to compute spatial derivatives of $f$ by analytic differentiation of the series. The linear map $\mathscr{D}_{N}$, which takes a vector of $N$ function values $\left\{f\left(x_{i}\right)\right\}$ to a vector of $N$ derivative values $\left\{f^{\prime}\left(x_{i}\right)\right\}$, is known as the spectral differentiation matrix. The precise form of $\mathscr{D}_{N}$ depends on the location of the points $\left\{x_{i}\right\}$ and the choice of the approximating series. For periodic functions, Fourier series are used with the function tabulated at equispaced nodes. For bounded domains, Chebyshev (or Legendre) series are used with the function tabulated at Chebyshev (or Legendre) nodes or extreme points.

Although spectral differentiation is remarkably accurate in exact arithmetic, there are a number of difficulties associated with its use. Ill-conditioning of the matrix with increasing $N$ frequently causes degradation of the observed precision. Furthermore, as recently demonstrated by Trefethen and Trummer for certain problems [12], [13], the time step restrictions due to this ill-conditioning can be more severe than those predicted by the standard stability theory.

In this paper, we will consider only the simplest steady-state case, namely linear two-point boundary value problems with constant coefficients. It is well known that problems of this type are efficiently and accurately solved by spectral methods. On the other hand, it is also well known that care must be taken in applying spectral methods to such problems. The naive approach leads to the use of unstable recurrence relations for the determination of the expansions coefficients, a consequence of the illconditioning of $\mathscr{D}_{N}$. Previous stable methods have consisted of a reformulation of the linear system by analytic or algebraic means [4], [5], [7], [8]. While avoiding the error amplification of the unstable recurrence relations in the calculation of the solution itself, information about derivatives, which is often of physical interest, still requires spectral differentiation.

Our main purpose in this paper is to suggest that well-conditioned spectral methods be developed through the use of integral equations. For two-point boundary value problems, this is accomplished by using the indefinite integral (and Clenshaw-Curtis

\footnotetext{
* Received by the editors August 14, 1989; accepted for publication (in revised form) August 31, 1990. The work of this author was supported in part by the U.S. Office of Naval Research under grant N00014-86-K0310 and in part by IBM under grant P00038437 and in part by a National Science Foundation Mathematical Sciences Postdoctoral Fellowship.

† Courant Institute of Mathematical Sciences, New York University, New York, New York 10012.
} 
quadrature [2]) to recast the governing differential equation as an integral equation for the second derivative, which is solved with spectral accuracy. The solution and first derivative may then be (stably) recovered by integration.

2. Chebyshev approximation. We will require several results from approximation theory. The Chebyshev polynomial of degree $k$ on $[-1,1]$ is defined by the formula

$$
T_{k}(\cos \theta)=\cos (k \theta) .
$$

Clearly, $\left|T_{k}(x)\right| \leqq 1$ for $x \in[-1,1]$,

$$
T_{0}(x)=1, \quad T_{1}(x)=x
$$

and, using elementary trigonometric identities,

$$
T_{k+1}(x)=2 x T_{k}(x)-T_{k-1}(x) \text { for } k \geqq 1 .
$$

The functions $T_{k}$ constitute an orthonormal basis with respect to the inner product

$$
(f, g)=\int_{-1}^{1} f(x) g(x)\left(1-x^{2}\right)^{-1 / 2} d x
$$

The Chebyshev nodes $t_{i}$ of degree $k$ are the zeros of $T_{k}$, namely,

$$
t_{i}=\cos \frac{(2 i+1) \pi}{2 k} \text { for } i=0,1,2, \cdots, k-1 \text {. }
$$

Let $C^{n}[-1,1]$ denote the set of functions defined on $[-1,1]$ with $n$ continuous derivatives. If $f \in C^{n}[-1,1]$ and

$$
\begin{aligned}
g(x) & =\frac{1}{2} a_{0} T_{0}(x)+a_{1} T_{1}(x)+a_{2} T_{2}(x)+\cdots \\
& =\sum_{k=0}^{\infty} a_{k} T_{k}(x)
\end{aligned}
$$

is the Chebyshev expansion associated with $f$, then

$$
a_{k}=\frac{2}{\pi} \int_{-1}^{1} f(x) T_{k}(x)\left(1-x^{2}\right)^{-1 / 2} d x=\frac{2}{\pi} \int_{0}^{\pi} f(\cos \theta) \cos k \theta d \theta .
$$

Moreover, the remainder in truncating the series at $N$ terms is of the order

$$
O\left(\frac{1}{N^{n-1}}\right) \text { as } N \rightarrow \infty \text {. }
$$

In particular, if $f$ is infinitely differentiable, then the remainder goes to zero superalgebraically (faster than any finite power of $1 / N$ ). For a more complete discussion, see Gottlieb and Orszag [7].

Remark 2.1. The introduction of the notation $\Sigma^{\prime}$ in (7) to represent the sum in (6) simplifies many of the formulas to follow.

Remark 2.2. In practice, the Chebyshev series (6) is truncated at some finite number of terms, say $N$. By relation (8), the coefficients $a_{k}$ are the coefficients of the Fourier cosine series of $F(\theta)=f(\cos \theta)$. Thus, if $f$ is tabulated at equispaced points in $\theta$, a condition satisfied by the Chebyshev nodes $\left\{t_{i}\right\}$, we can obtain all $N$ coefficients $a_{k}$ by means of the Fast Fourier Transform (FFT) or, more precisely, the Fast Cosine Transform, using $O(N \log N)$ operations. Similarly, the inverse cosine transform can be used to compute function values $g(x) \approx f(x)$ at the nodes $\left\{t_{i}\right\}$ from the coefficients $a_{k}$ of the expansion. 
Remark 2.3. In certain calculations it is useful to use a set of discrete nodes which includes one or both endpoints of the interval $[-1,1]$, such as the Gauss-Lobatto points [3]. Such a choice does not affect the obtained results and we will consider only the classical "Chebyshev" nodes in our discussion.

\subsection{Differentiation and integration of Chebyshev expansions.}

Definition 2.1. Let $X$ be the space consisting of infinite sequences of real numbers

$$
\mathbf{x}=\left(x_{0}, x_{1}, x_{2}, \cdots\right) \text {. }
$$

For any $\mathbf{a} \in X$, we will denote by $\mathscr{D}(\mathbf{a})$ the sequence $\mathbf{b}$ given by the formula

$$
b_{k}=\sum_{\substack{p=k+1 \\ p+k \text { odd }}}^{\infty} p \cdot a_{p} .
$$

We will refer to the mapping $\mathscr{D}: X \rightarrow X$ as spectral differentiation. We will denote by $\mathscr{I}(a)$ the sequence $\mathbf{d}$ given by the formulae

$$
\begin{gathered}
d_{k}=\frac{1}{2 k}\left(a_{k-1}-a_{k+1}\right) \quad \text { for } k \geqq 1, \\
d_{0}=d_{1}-d_{2}+d_{3}-\cdots .
\end{gathered}
$$

We will refer to the mapping $\mathscr{I}: X \rightarrow X$ as spectral integration.

The definitions above are motivated by the following lemma, which may be found in the Appendix to [7].

LEMMA 2.1. Let $f$ be a smooth function given by a Chebyshev series

$$
f(x)=\sum_{k=0}^{\infty} a_{k} T_{k}(x) .
$$

Then the derivative of $f$ has a series expansion of the form

$$
f^{\prime}(x)=\sum_{k=0}^{\infty} b_{k} T_{k}(x)
$$

with $b_{k}$ given by (10). The integral of $f$ has a series expansion of the form

$$
\int_{-1}^{x} f(t) d t=\sum_{k=0}^{\infty} d_{k} T_{k}(x),
$$

where $d_{k}$ is given by (11) and (12).

Remark 2.4. The series expansion (15) is the basis for Clenshaw-Curtis quadrature [2]. After obtaining the coefficients $d_{k}$, we compute

$$
\int_{-1}^{1} f(x) d x=2 \sum_{k=0}^{\infty} d_{2 k+1},
$$

which follows immediately from the equalities

$$
T_{k}(-1)=(-1)^{k} \text { and } T_{k}(1)=1 .
$$

It is clear from (10)-(12) that $\mathscr{D}$ is unbounded in the $l_{\infty}$ norm, while $\mathscr{I}$ is bounded. This behavior is reflected in the numerical conditioning of the finite-dimensional analogues of these operators. If $f$ is represented by a truncated Chebyshev expansion

$$
f(x)=\sum_{k=0}^{N} a_{k} T_{k}(x)
$$


then the coefficients of $f^{\prime}$ are still given by (10), but the summation is truncated at $N$ terms. Now let $\mathbf{a}=\left(a_{0}, a_{1}, \cdots, a_{N}\right), \hat{\mathbf{a}}=\left(a_{0}+\varepsilon, a_{1}+\varepsilon, \cdots, a_{N}+\varepsilon\right), \mathscr{D}(\mathbf{a})=\mathbf{b}$, and $\mathscr{D}(\hat{\mathbf{a}})=\hat{\mathbf{b}}$. Then

$$
\frac{\|\hat{\mathbf{b}}-\mathbf{b}\|_{\infty}}{\|\hat{\mathbf{a}}-\mathbf{a}\|_{\infty}}=O\left(N^{2}\right),
$$

the maximum relative error being incurred for the calculation of $b_{0}$. In other words, the process of differentiation via Chebyshev series can amplify errors by a factor proportional to $N^{2}$. On the other hand, it is easy to show that for any $\hat{\mathbf{a}}$,

$$
\frac{\left|\hat{\mathbf{d}}_{k}-\mathbf{d}_{k}\right|}{\|\hat{\mathbf{a}}-\mathbf{a}\|_{\infty}} \leqq 1 \quad \text { for } k \geqq 1,
$$

where $\mathbf{d}=\mathscr{I}(\mathbf{a})$ and $\hat{\mathbf{d}}=\mathscr{I}(\hat{\mathbf{a}})$. Using the definitions (11) and (12), an elementary calculation shows that

$$
\frac{\left|\hat{\mathbf{d}}_{0}-\mathbf{d}_{0}\right|}{\|\hat{\mathbf{a}}-\mathbf{a}\|_{\infty}} \leqq \frac{1}{2}+\frac{1}{4}+\zeta(2)<2.4,
$$

where $\zeta$ denotes the Riemann zeta function. In other words, the process of integration via Chebyshev series amplifies errors by a factor of less than 2.4 .

2.2. The spectral integration matrix. The spectral differentiation matrix for Chebyshev nodes can be expressed in terms of $\mathscr{D}$ by the formula

$$
\mathscr{D}_{N}=\mathscr{C}_{N}^{-1} \cdot \mathscr{D} \cdot \mathscr{C}_{N} \text {, }
$$

where $\mathscr{C}_{N}$ is the discrete cosine transform of dimension $N$.

Definition 2.2. The spectral integration matrix for Chebyshev nodes $\mathscr{I}_{N}$ is defined by the formula

$$
\mathscr{I}_{N}=\mathscr{C}_{N}^{-1} \cdot \mathscr{I} \cdot \mathscr{C}_{N}
$$

It is clear that the matrix $\mathscr{I}_{\mathcal{N}}$ can be applied to a vector in $O(N \log N)$ operations by using a Fast Cosine Transform algorithm.

Before turning to the solution of two-point boundary value problems, we briefly investigate the behavior of $\mathscr{D}_{\mathcal{N}}$ and $\mathscr{I}_{\mathcal{N}}$ with a set of three examples (Fig. 1). Note that in these examples, we test $\mathscr{D}_{\mathcal{N}}$ by differentiating a function $f(x)$ and we test $\mathscr{I}_{\mathcal{N}}$ by integrating the corresponding derivative $f^{\prime}(x)$. The integration of $f(x)$ itself is even more stable and of less interest. When $f(x)=\sin (x)$, we observe the expected convergence as soon as the number of sampling points is sufficient, approximately two points per wavelength at the coarsest part of the grid. However, as the number of points increases, differentiation becomes less and less accurate while integration is essentially unaffected. Although there is no need to use 1,000 points to resolve $\sin (x)$ alone, the error introduced by its differentiation remains when the problem becomes more complex and more points are required. For example, when $f(x)=\sin (x)+0.01 \sin (10 x)$, about 50 points are required to achieve spectral accuracy, at which point an error of the order $10^{-4}$ has already been incurred. When $f(x)=\sin (x)+0.005 \sin (60 x)$, the situation is worse. In single precision, even with the optimal choice of $N$, the mean square error is greater than 1 percent.

3. Two-point boundary value problems. The two-point boundary value problems considered here are second-order equations of the form

$$
L u=u^{\prime \prime}+\mu u^{\prime}+\nu u=f(x), \quad x \in[-1,1]
$$



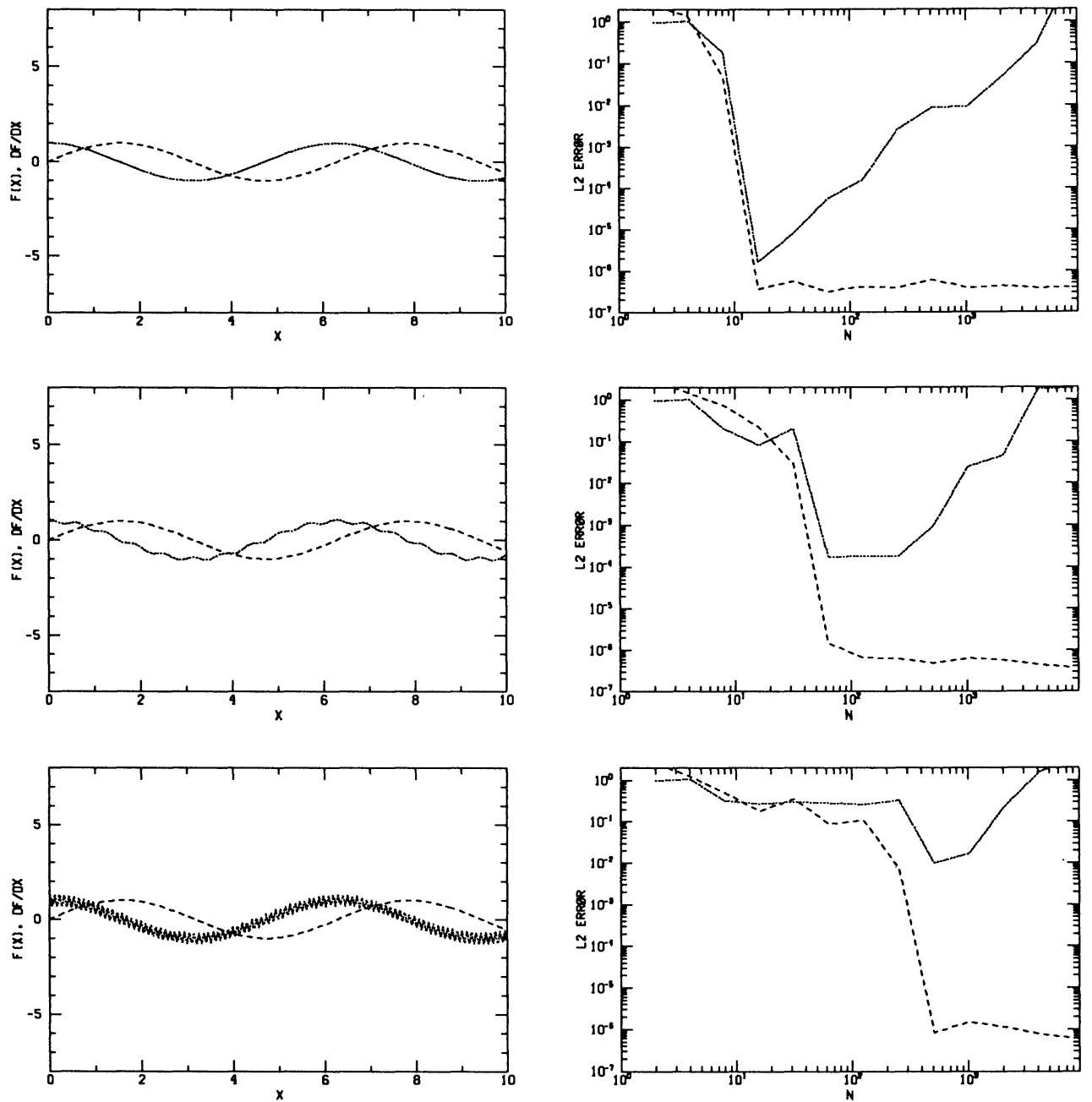

FIG. 1. The numerical behavior of spectral differentiation and integration is demonstrated with three examples. The left hand of each pair of figures is a plot of the exact functions $f(x)$ as a dashed curve and $f^{\prime}(x)$ as a dotted curve. The right hand of each pair is a plot of the mean square error in the spectral differentiation of $f(x)$ (dotted line) and the spectral integration of $f^{\prime}(x)$ (dashed line) versus the number of points in the discretization. For the top figure $f(x)=\sin (x)$, for the middle figure $f(x)=\sin (x)+0.01 \sin (10 x)$, and for the bottom figure $f(x)=\sin (x)+0.005 \sin (60 x)$. Calculations were done in single precision.

with $\mu, \nu \in \mathbb{R}$ and Dirichlet conditions

$$
u(-1)=\alpha, \quad u(1)=\beta .
$$

The fact that high-order polynomial approximations achieve superalgebraic convergence for such differential equations has been known for a long time. Ciarlet, Schultz, and Varga [1] have shown that superalgebraic convergence can be achieved even when the governing ordinary differential equation is nonlinear, so long as the solution is sufficiently smooth. In the standard spectral formulation [7], we seek a solution

$$
u_{N}(x)=\sum_{k=0}^{N} a_{k} T_{k}(x),
$$


subject to the boundary conditions

$$
\sum_{k=0}^{N}(-1)^{k} a_{k}=\alpha \quad \text { and } \quad \sum_{k=0}^{N} a_{k}=\beta,
$$

where we have used the equalities in (17). From the differential equation (24) and the spectral differentiation matrix, we obtain the relations

$$
\sum_{\substack{p=k+2 \\ p+k \text { even }}}^{N} p\left(p^{2}-k^{2}\right) a_{p}+\mu \sum_{\substack{p=k+1 \\ p+k \text { odd }}}^{N} p a_{p}+\nu a_{k}=f_{k} \text { for } k=0, \cdots, N-2,
$$

where the $\left\{f_{k}\right\}$ are the Chebyshev expansion coefficients for the right-hand side $f(x)$. This set of equations is inherently ill-conditioned. A reformulation of the recurrence relations is, therefore, used to compute the solution in a stable manner (see [7, p. 119]).

Consider now the one-dimensional Poisson equation

$$
u^{\prime \prime}=f(x), \quad u(-1)=\alpha, \quad u(1)=\beta .
$$

Rather than setting up the recurrence relations as in (28), it would be very attractive to be able to write

$$
u(x)=\int_{-1}^{x} \int_{-1}^{t} f(\tau) d \tau d t+C_{1} x+C_{0} .
$$

The spectral integration matrix of Definition 2.1 provides us with precisely the ability to compute this formal solution. The constants $C_{1}$ and $C_{0}$ are chosen to satisfy the boundary condition.

When the differential equation contains terms in $u^{\prime}$ or $u$, we still have a simple analytic expression for the solution. We may assume, without loss of generality, that we are given homogeneous boundary conditions and that the corresponding Green's function has the form

$$
G(x, t)= \begin{cases}u_{1}(x) v_{1}(t) & \text { for } x<t \\ u_{2}(x) v_{2}(t) & \text { for } x>t,\end{cases}
$$

where $u_{1}$ and $u_{2}$ are solutions of the homogeneous equation $L u=0$. Since the equation has constant coefficients, $u_{1}$ and $u_{2}$ are known explicitly. The desired solution can then be written as

$$
\begin{aligned}
u(x)= & \int_{-1}^{1} G(x, t) f(t) d t \\
= & u_{1}(x) \cdot \int_{-1}^{x} v_{1}(t) f(t) d t \\
& \quad+u_{2}(x) \cdot\left(\int_{-1}^{1} v_{2}(t) f(t) d t-\int_{-1}^{x} v_{2}(t) f(t) d t\right) .
\end{aligned}
$$

The indefinite integrals in the preceding expression can be tabulated by means of the spectral integration matrix, while the definite integral can be computed by formula (16). Once this initial work, requiring two Fast Cosine Transforms, is done, the solution is obtained using approximately $3 N$ additional operations, where $N$ is the number of Chebyshev nodes used. A similar observation is made by Rokhlin in [9], where the integrals are computed by a finite-order quadrature formula. His approach has the advantage that it does not depend on the location of the discretization nodes. One 
drawback of this particular use of Green's functions is that the terms $u_{1}, v_{1}, u_{2}$, and $v_{2}$ may behave much more violently than either the right-hand side or the solution, requiring many more points in the discretization than otherwise necessary. (Consider, e.g., the equation $y^{\prime \prime}-\alpha y=f$ for large values of $\alpha$.) Another drawback is that the method does not extend to nonconstant coefficient problems, since we cannot determine the Green's function analytically.

We consider a closely related approach which does not require knowledge of the Green's function. An integral equation is constructed by solving for $\sigma(x)=u^{\prime \prime}(x)$ rather than $u$ itself. The original system (24) becomes

$$
\sigma(x)+\mu \int_{-1}^{x} \sigma(t) d t+\mu C_{1}+\nu \int_{-1}^{x} \int_{-1}^{t} \sigma(\tau) d \tau d t+\nu C_{1} x+\nu C_{0}=f(x) .
$$

Representing $\sigma(x)$ and $f(x)$ by truncated Chebyshev series

$$
\sigma(x)=\sum_{k=0}^{N} a_{n} T_{n}(x), \quad f(x)=\sum_{k=0}^{N} f_{n} T_{n}(x)
$$

and using the spectral integration matrix, we obtain the system of equations

$$
\begin{aligned}
& a_{0}+\mu C_{1}+\nu C_{0}=f_{0} \\
& a_{1}+\frac{\mu}{2}\left(a_{0}-a_{2}\right)+\frac{\nu}{8}\left(8 C_{1}+a_{1}-a_{3}\right)=f_{1} \\
& \ldots \\
& a_{k}+\frac{\mu}{2 k}\left(a_{k-1}-a_{k+1}\right)+\frac{\nu}{2 k}\left(\frac{1}{2 k-2}\left(a_{k-2}-a_{k}\right)-\frac{1}{2 k+2}\left(a_{k}-a_{k+2}\right)\right)=f_{k} \\
& \quad \text { for } k=2, \cdots, N .
\end{aligned}
$$

This is a sytem of $N+1$ equations with $N+3$ unknowns (the coefficients $a_{k}$ and the constants of integration $C_{0}$ and $C_{1}$ ). Two additional equations are obtained from the boundary conditions

$$
\begin{gathered}
C_{0}-C_{1}+\sum_{k=2}^{N} \frac{1}{2 k}\left(\frac{1}{2 k-2}\left(a_{k-2}-a_{k}\right)-\frac{1}{2 k+2}\left(a_{k}-a_{k+2}\right)\right)(-1)^{k}=\alpha, \\
C_{0}+C_{1}+\sum_{k=2}^{N} \frac{1}{2 k}\left(\frac{1}{2 k-2}\left(a_{k-2}-a_{k}\right)-\frac{1}{2 k+2}\left(a_{k}-a_{k+2}\right)\right)=\beta .
\end{gathered}
$$

The discrete problem is pentadiagonal except for the two rows derived from the boundary conditions, and can be solved using approximately $10 \mathrm{~N}$ floating point operations.

4. Numerical examples. A two-point boundary value problem solver, using the method of the previous section, has been implemented and tested on a variety of examples. It requires two cosine transforms and the solution of one linear system, with a total computational cost estimated at $10 N(\log N+1)$ floating point operations. All calculations cited below were carried out in double precision on a SUN 3/50 workstation with f68881 floating point accelerator.

The behavior of the algorithm is demonstrated by three examples (Fig. 2 and Table 1). In the first case, we used a model problem from Stoer and Bulirsch [11]:

$$
\begin{gathered}
-y^{\prime \prime}+400 y=-400 \cos ^{2} \pi x-2 \pi^{2} \cos 2 \pi x, \\
y(0)=y(1)=0,
\end{gathered}
$$



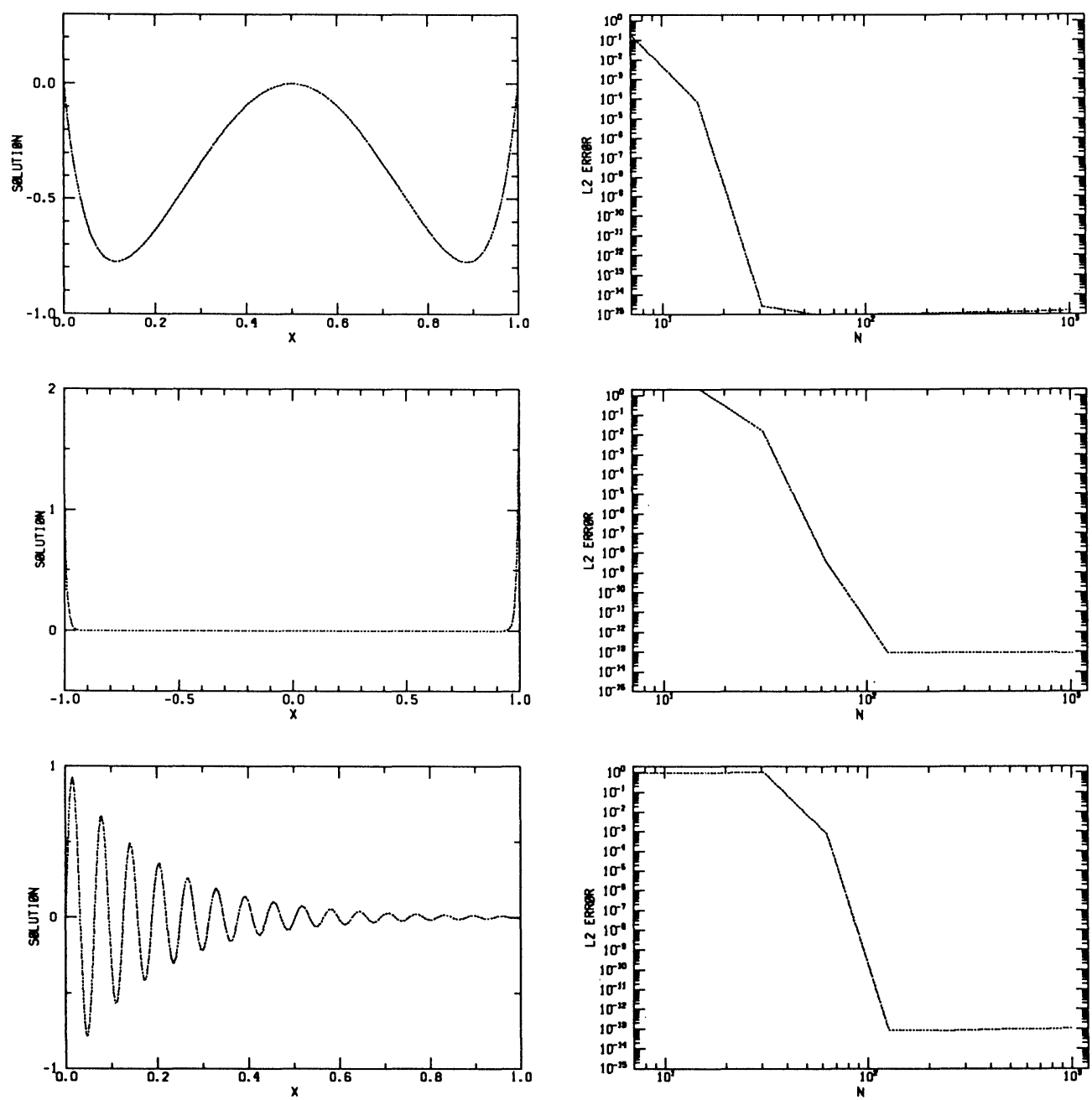

FIG. 2. The numerical behavior of the integral equation algorithm, using three examples. The left hand of each pair of figures is a plot of the exact solution as a function of $x$. The right hand of each pair is a plot of the mean square error in the computed solution versus the number of points in the discretization. The governing differential equations are discussed in the text.

TABLE 1

CPU times (in seconds) and mean square errors in computing the solutions to three boundary value problems. The subscripts 1-3 refer to the three equations discussed in the text and displayed graphically in Fig. 2 from top to bottom.

\begin{tabular}{|c|c|c|c|c|c|c|}
\hline$N$ & $T_{1}$ & $E_{1}$ & $T_{2}$ & $E_{2}$ & $T_{3}$ & $E_{3}$ \\
\hline 16 & 0.08 & $7.2 \times 10^{-5}$ & 0.06 & 2.2 & 0.06 & 0.9 \\
\hline 64 & 0.30 & $8.7 \times 10^{-16}$ & 0.32 & $4.0 \times 10^{-9}$ & 0.32 & $7.9 \times 10^{-4}$ \\
\hline 256 & 1.46 & $1.1 \times 10^{-15}$ & 1.48 & $9.1 \times 10^{-14}$ & 1.40 & $8.1 \times 10^{-14}$ \\
\hline 1,024 & 6.42 & $1.5 \times 10^{-15}$ & 6.32 & $9.1 \times 10^{-14}$ & 6.10 & $1.0 \times 10^{-13}$ \\
\hline
\end{tabular}


with exact solution

$$
y(x)=\frac{e^{-20}}{1+e^{-20}} e^{20 x}+\frac{1}{1+e^{-20}} e^{-20 x}-\cos ^{2} \pi x .
$$

Standard finite-difference and finite-element methods tend to converge quite slowly, due to the large derivatives of the exact solution near the boundaries. While multiple shooting, which is recommended in [11], is a viable approach, the method is computationally expensive. Our calculations show that the corresponding integral equation is solved to spectral accuracy with very little effort.

The second example involves a boundary layer near each endpoint. The governing equation is

$$
\begin{gathered}
\varepsilon y^{\prime \prime}-y=0, \\
y(-1)=1, \quad y(1)=2,
\end{gathered}
$$

where $\varepsilon=10^{-5}$. As is well known, the Chebyshev nodes are particularly good at resolving boundary layers since they tend to cluster at the two endpoints (see [7]).

The third example is one where the solution is very oscillatory:

$$
\begin{gathered}
y^{\prime \prime}+5 y^{\prime}+10,000 y=-500 \cos (100 x) e^{-5 x}, \\
y(0)=0, \quad y(1)=\sin (100) e^{-5},
\end{gathered}
$$

for which the exact solution is

$$
y(x)=\sin (100 x) e^{-5 x} .
$$

In each case, it is clear that the spectral integral formulation is both rapidly convergent and stable.

5. Conclusions. The spectral integration matrix is a well-conditioned operator which yields an antiderivative of a function tabulated at Chebyshev nodes. In this paper, we have presented a fast algorithm for the solution of constant coefficient two-point boundary value problems through the use of integral equations and spectral integration. The central advantage of the scheme proposed in this paper over the stabilized spectral approach [7, p. 119] is that we can obtain first and second derivatives of the solution without the instability of differentiation. These derivatives are needed in many calculations of practical interest.

The difficulty with variable coefficient problems lies not in the formulation of the integral equation, but in the fact that the resulting system of equations for the coefficients of the Chebyshev series of $u^{\prime \prime}$ is dense. Gaussian elimination would require $O\left(N^{3}\right)$ operations, where $N$ is the number of Chebyshev nodes used in the discretization. On the other hand, the spectral integration matrix can be used to apply the integral operator in $O(N \log N)$ operations, making iterative methods more attractive (see, e.g., [9]). The number of iterations required is a function of the underlying problem, and does not increase with the number of nodes. Unfortunately, for many situations of interest, complex behavior of the solution causes the condition number of the underlying problem and the number of iterations to be large, so that direct methods would be preferable provided that an $O(N)$ or $O(N \log N)$ operation count could be maintained. Recently, such algorithms have been designed, both for second-order differential equations [6] and more general first-order systems [10]. 
Acknowledgments. The author thanks V. Rokhlin, F. Saied, and L. N. Trefethen for many useful discussions.

Note added in proof. A. Zebib has investigated issues similar to those addressed here and arrived at similar conclusions [14]. We would like to thank D. Henningson for bringing this paper to our attention.

\section{REFERENCES}

[1] P. G. Ciarlet, M. H. Schultz, AND R. S. VARGa, Numerical methods of high-order accuracy for nonlinear boundary value problems I. One dimensional problem, Numer. Math., 9 (1967), pp. 394-430.

[2] C. W. Clenshaw AND A. R. Curtis, A method for numerical integration on an automatic computer, Numer. Math., 2 (1960), pp. 197-205.

[3] P. J. Davis And P. Rabinowitz, Methods of Numerical Integration, Academic Press, Orlando, FL, 1984.

[4] S. C. R. DENNIS AND L. QUARTAPELle, Spectral algorithms for vector elliptic equations in a spherical gap, J. Comput. Phys., 9 (1985), pp. 218-241.

[5] L. Fox AND I. B. PARKer, Chebyshev Polynomials in Numerical Analysis, Oxford University Press, London, 1968.

[6] L. Greengard AND V. Rokhlin, On the numerical solution of two-point boundary value problems, Comm. Pure Appl. Math., to appear.

[7] D. GotTlieb And S. Orszag, Numerical Analysis of Spectral Methods, Society for Industrial and Applied Mathematics, Philadelphia, PA, 1977.

[8] C. Lanczos, Applied Analysis, Prentice-Hall, Englewood Cliffs, NJ, 1956.

[9] V. Rokhlin, Application of volume integrals to the solution of partial differential equations, Comput. Math. Appl., 11 (1985), pp. 667-679.

[10] P. STARR AND V. RoKHLIN, On the numerical solution of two-point boundary value problems II, Research Report 802, Dept. of Computer Science, Yale University, New Haven, CT, 1990.

[11] J. Stoer AND R. Bulirsch, Introduction to Numerical Analysis, Springer-Verlag, New York, 1980.

[12] L. N. Trefethen, Lax-stability vs. eigenvalue stability of spectral methods, in Numerical Methods for Fluid Dynamics III, K. W. Morton and M. J. Baines, eds., Clarendon Press, Oxford, 1988.

[13] L. N. Trefethen AND M. R. Trummer, An instability phenomenon in spectral methods, SIAM J. Numer. Anal., 24 (1987), pp. 1008-1023.

[14] A. ZEBIB, A Chebyshev method for the solution of boundary value problems, J. Comput. Phys., 53 (1984), pp. 443-455. 